\title{
Comparative metabolomics in vegans and omnivores reveal constraints on diet-dependent gut microbiota metabolite production
}

\author{
Gary D Wu, ${ }^{1}$ Charlene Compher, ${ }^{2}$ Eric Z Chen, ${ }^{3}$ Sarah A Smith, ${ }^{1}$ Rachana D Shah, ${ }^{4}$ \\ Kyle Bittinger, ${ }^{5}$ Christel Chehoud, ${ }^{5}$ Lindsey G Albenberg, ${ }^{6}$ Lisa Nessel, ${ }^{3}$ Erin Gilroy, ${ }^{3}$ \\ Julie Star, ${ }^{1}$ Aalim M Weljie, ${ }^{7}$ Harry J Flint, ${ }^{8}$ David C Metz, ${ }^{1}$ Michael J Bennett, ${ }^{9}$ \\ Hongzhe Li, ${ }^{3}$ Frederic D Bushman, ${ }^{5}$ James D Lewis ${ }^{1,3}$
}

\begin{abstract}
- Additional material is published online only. To view please visit the journal online (http://dx.doi.org/10.1136/ gutjnl-2014-308209)

For numbered affiliations see end of article.
\end{abstract}

\section{Correspondence to}

Gary D Wu, 915 BRB II/III, 421

Curie Blvd, Perelman School of

Medicine, University of

Pennsylvania, Philadelphia,

PA 19104;

gdwu@mail.med.upenn.edu

James D Lewis, 720 Blockley Hall, 423 Guardian Drive,

Philadelphia, PA 19104-6021;

lewisjd@mail.med.upenn.edu

Frederic D Bushman, 426 Johnson Pavilion, Department of Microbiology, 3610

Hamilton Walk, Philadelphia, PA 19104-6076; bushman@ mail.med.upenn.edu

Received 6 August 2014 Revised 28 October 2014 Accepted 29 October 2014 Published Online First 27 November 2014

\section{CrossMark}

To cite: Wu GD

Compher $\mathrm{C}$, Chen $\mathrm{EZ}$, et al. Gut 2016:65:63-72.

\section{ABSTRACT}

Objective The consumption of an agrarian diet is associated with a reduced risk for many diseases associated with a 'Westernised' lifestyle. Studies suggest that diet affects the gut microbiota, which subsequently influences the metabolome, thereby connecting diet, microbiota and health. However, the degree to which diet influences the composition of the gut microbiota is controversial. Murine models and studies comparing the gut microbiota in humans residing in agrarian versus Western societies suggest that the influence is large. To separate global environmental influences from dietary influences, we characterised the gut microbiota and the host metabolome of individuals consuming an agrarian diet in Western society.

Design and results Using 165 rRNA-tagged sequencing as well as plasma and urinary metabolomic platforms, we compared measures of dietary intake, gut microbiota composition and the plasma metabolome between healthy human vegans and omnivores, sampled in an urban USA environment. Plasma metabolome of vegans differed markedly from omnivores but the gut microbiota was surprisingly similar. Unlike prior studies of individuals living in agrarian societies, higher consumption of fermentable substrate in vegans was not associated with higher levels of faecal short chain fatty acids, a finding confirmed in a 10-day controlled feeding experiment. Similarly, the proportion of vegans capable of producing equol, a soy-based gut microbiota metabolite, was less than that was reported in Asian societies despite the high consumption of soy-based products.

Conclusions Evidently, residence in globally distinct societies helps determine the composition of the gut microbiota that, in turn, influences the production of diet-dependent gut microbial metabolites.

\section{INTRODUCTION}

Among the many factors that influence the gut microbiota, diet has received considerable attention because of its potential impact on health. Studies using rodent models, surveys in various mammalian species and cross-sectional studies in globally distinct human populations suggest that diet has a strong effect on the composition of the gut microbiota. $^{1-5}$ By contrast, more moderate dietary

\section{Significance of this study}

What is already known on this subject?

- The consumption of a plant-based agrarian diet is associated with health benefits.

- Diet alters the composition of the gut microbiota and serves as a substrate for bacterial metabolism that could influence health of the host.

- Data from murine models and gut microbiota in humans residing in agrarian versus Western societies suggest that the influence of diet on the composition of the gut microbiota is large.

What are the new findings?

- The impact of diet on the plasma metabolome of omnivores and vegans is large but its effect on the composition of the gut microbiota is surprisingly modest.

- Gut microbiota metabolites contribute more to the plasma metabolome of vegans than omnivores.

- The production of gut bacteria-derived metabolites from dietary substrates is constrained by the composition of the gut microbiota.

How might it impact on clinical practice in the foreseeable future?

- Environmental factors independent of diet may play a critical role in shaping the composition of the gut microbiota in globally distinct human societies, which, in turn, has an effect on the production of beneficial metabolites such as short chain fatty acids and equol from diet and the gut microbiota.

- Development of prebiotics to deliver substrates for the gut microbiota to produce desirable metabolites that will favour health must take into consideration the composition of the gut microbiota.

interventions that can be sustained in humans long term suggest that the impact of diet may be more modest. $^{6-8}$ Long-term consumption of agrarian plant-based diets has been associated with greater 
taxonomic and bacterial gene diversity, higher levels of short chain fatty acid production and greater Prevotella/Bacteroides ratio. ${ }^{2}$ Multiple disease states are associated with a decrease in the diversity of the gut microbiota, a hallmark of 'dysbiosis'thus reversal of the dysbiotic microbiota through dietary modification or other means could serve to maintain health and/or treat disease.

Diet provides the substrates for the production of a multitude of small molecules that, after first-pass metabolism in the liver, circulate systemically where they may have diverse effects on host physiology. ${ }^{9}$ For example, dietary choline or carnitine can be converted by the gut microbiota to trimethyl amine that is subsequently oxidised in the liver to trimethyl amine oxide, a small molecule associated with increased risk for coronary vascular disease. ${ }^{10}{ }^{11}$ With a genome size approximately 150-fold greater than the mammalian host, the metabolome of the gut microbiota allows production of a multitude of small molecules not produced by the host. ${ }^{12}$ Specific molecules produced are regulated by substrate availability, product abundance, microbial gene activity and other mechanisms not readily inferred from the simple quantification of microbial gene abundance. Thus, taxonomic information of the gut microbiota, or even whole genome analysis, may not be adequate to predict the metabolome of a complex microbial community.

Here, we studied healthy vegans and omnivores to investigate the effect of diet on the gut microbiota and the host metabolome in subjects living in an urban Western environment. We observed differences between the groups in macronutrient and micronutrient consumption, the composition of the gut microbiota, and plasma and urinary metabolomes. Differences in the metabolome largely reflected the lower intake of both protein and fat and higher intake of carbohydrates in vegans. The vegan metabolome contained a higher level of metabolites produced by the gut microbiota. In contrast to diet, the microbiota composition was not strongly associated with plasma metabolite levels. Similarly, there was a surprisingly modest difference in the composition of the gut microbiota between omnivores and vegans. The similarity in the taxonomy of the gut microbiota despite the divergent diets allowed us to determine the impact of substrate abundance versus bacterial community membership on the production of two well-characterised products of gut microbiota metabolism, short chain fatty acids (SCFAs) and equol. Our analyses demonstrate that dietary delivery of substrates to the gut microbiota is necessary but not sufficient to control product generation-metabolites were only produced when specific bacterial lineages were also present.

\section{MATERIALS AND METHODS \\ Human subjects}

Exclusion criteria have been previously described for our crosssectional study among vegans and omnivores and longitudinal controlled feeding experiment (CAFÉ) among omnivores. ${ }^{6}$ The cross-sectional study included 15 vegans and six omnivores who were newly recruited and the baseline data of 10 omnivores included in the CAFÉ. ${ }^{6}$ The vegans consumed a vegan diet for a minimum of 6 months. Each participant completed three $24 \mathrm{~h}$ dietary recalls within 1 week as previously described $^{6}$ after which faecal, fasting blood and urine samples were collected.

\section{S rRNA gene sequencing and plasma metabolomics}

DNA was isolated from stool as described in refs. 6 and 13. Bacterial 16S rRNA gene sequences were PCR amplified using primers binding to the V1V2 region ${ }^{6}{ }^{13}$ using bar-coded primers. ${ }^{14} 15$ Sequence reads were quality controlled and analysed using the QIIME pipeline with default parameters. ${ }^{16}$ Human plasma samples were extracted and analysed on liquid chromatography/mass spectrometry (LC/MS), LC/MS/MS and gas chromatography (GC)/MS platforms by Metabolon (Durham, North Carolina, USA).

\section{Plasma protein assays}

Plasma glucose, cholesterol, high-density lipoprotein (HDL) and triglycerides were determined on a COBAS c501 (Roche, Indianapolis, Indiana, USA); estimated low-density lipoprotein (LDL) was calculated using the equation ( $\mathrm{LDL}=$ total cholesterol-HDL cholesterol(triglycerides $\div 5)$ ). Plasma insulin was measured by radioimmunoassay with intra-assay coefficient of variation (CV) of $2.2 \%$. Plasma adipokines and cytokines were measured by ELISA (R\&D Systems, Minneapolis, Minnesota, USA).

\section{Urinary metabolomics}

Analysis of organic acids by analysis of trimethylsilyl derivatives of ethylacetate extracted organic acids was performed using GC electron impact MS as previously described. ${ }^{17}$ Data were accumulated by full scan ion collection over the range 50-600 m/z. Peak identities were confirmed by National Institute of Standards and Technology (NIST) library search.

\section{Faecal SCFA analysis}

Nuclear magnetic resonance (NMR) spectra of faecal water were acquired using nuclear Overhauser spectroscopy (NOESY) presaturation on a four-channel Bruker Ascend $700 \mathrm{MHz}$ spectrometer (Bruker, Germany), and a 'targeted profiling' approach $^{18}$ of quantitatively characterising NMR spectra was applied where concentrations were quantitatively determined using the $700 \mathrm{MHz}$ library from Chenomx NMR Suite V.7.1 (Chenomx, Edmonton, Canada).

\section{Hydrogen-methane breath testing}

Hydrogen and methane production were quantified using methods similar to previously described methods. ${ }^{19}$ Briefly, after an overnight fast and baseline values determined, samples were obtained at $15 \mathrm{~min}$ intervals for $3 \mathrm{~h}$ following ingestion of $10 \mathrm{~g}$ of lactulose with gas analysis by Breathtracker gas chromatograph (Quintron, Milwaukee, Wisconsin).

\section{Bioinformatic and statistical analyses}

Micronutrient intake was standardised using linear regression adjusted for total calorie intake with residual values centred and scaled. Principal component analysis, multidimensional scaling (MDS) and permutational multivariate analysis of variance (PERMANOVA) analyses were performed in R. Weighted and unweighted UniFrac distances were used to compare overall microbiome composition between vegans and omnivores and were used for MDS analyses. Standardised micronutrient quantities and log-transformed metabolites between vegans and omnivores were compared by $\mathrm{t}$ test. Clustering analysis used Manhattan distances for the microbiota samples and correlation coefficients for micronutrients or metabolites. Wilcoxon rank-sum and Fisher's exact test identified differential bacterial genera abundance between vegans and omnivores. Random forest (RF) classification was used to evaluate the predictive accuracy of metabolite data to distinguish vegans from omnivores. Bacterial genus diversity was determined by Simpson's index. PICRUSt was used to infer gene representation using taxanomic information from 16S rRNA gene sequencing. ${ }^{20}$ 


\section{RESULTS}

\section{Macronutrient and micronutrient consumption in omnivores and vegans}

Dietary consumption was quantified using the mean of three 24 h dietary recalls. Data for one participant were excluded due to implausibly low-reported calorie intake. Consolidation of the 155 macronutrients and micronutrients into principle components revealed clear separation of the dietary intake between the omnivores and vegans (figure 1A). Vegans consumed more carbohydrates, 296.9 (98.3) g versus 246.5 (81.2) g, but less protein, 79.1 (22.9) g versus 89.1 (33) g and fat, 63.8 (20.5) g versus 86.3 (39.1) g than omnivores (figure 1B). Over half of the macronutrients and micronutrients were significantly different between groups (see online supplementary table S1) despite the relatively modest size of our sample set $(n=16$ for omnivores and $n=15$ for vegans).

\section{Only slight differences between the gut microbiota of omnivores and vegans.}

To characterise the microbiota of our subjects, we collected faecal samples, purified DNA and amplified and sequenced 16S rRNA gene tags (V1V2 region). Despite the distinctive diets (figure 1 and online supplementary table S1), 16S sequence data showed a modest, though statistically significant, difference in composition in unweighted (presence/absence) Unifrac analysis (figure 2A, PERMANOVA, $\mathrm{p}=0.007$ ), but not in an abundance weighted analysis (figure $2 \mathrm{~B}, \mathrm{p}=0.15$ ). Only seven taxa had differential presence at a nominal $\mathrm{p}$ value $<0.05$ (Fisher's exact test, see online supplementary table S2), all with median relative abundance $<1 \%$; Prevotella was not amongst these. ${ }^{6}$ No taxa differed significantly in the presence or abundance at the genus level after correction for multiple comparisons ( $q$ value, see online supplementary table S2). Measures of diversity and evenness were not significantly different between groups (figure 2C, Simpson index $\mathrm{p}=0.53$ ) nor was there any difference in phylogenetic diversity (see online supplementary figure S1). Thus, differences in gut microbiota between omnivores and vegans sampled in an urban environment in the Northeastern USA were quite modest.

\section{The plasma metabolome of omnivores is determined largely by diet.}

The health benefits of a plant-based diet have been well described. ${ }^{21}$ Our protocol excluded participants with immunodeficiency syndromes or significant GI diseases, but as a further check, biomarkers associated with metabolic diseases such as body mass index (BMI), glucose homeostasis, inflammatory markers and plasma lipid levels were compared between omnivores and vegans (see online supplementary figure S2). Only total cholesterol $(p=0.04)$ and LDL $(p=0.016)$ differed between the groups, with levels being higher in omnivores as expected.

The concentration of 92 plasma metabolites, of 361 metabolites tested (25\%), differed between omnivores and vegans (q value $<0.05$, figure $3 \mathrm{~A}$ and see online supplementary table S3). Thirty out of 144 lipid and 22 out of 88 amino acid metabolites, labelled yellow and red on the upper $\mathrm{x}$-axis, were significantly elevated in omnivores ( $q$ value $<0.05$, see online supplementary table S3). Eleven out of 47 xenobiotic metabolites, labelled purple, are significantly elevated in vegans (q value $<0.05$, see online supplementary table S3). Using RF classification, a multivariate technique reporting on the consensus of a large number of decision trees, we analysed the plasma metabolome to identify biochemicals partitioned in subjects according to diet. A model based on 30 metabolites, roughly categorised into six areas (amino acids, carbohydrates, cofactors and vitamins, lipids, nucleotides and xenobiotics) resulted in a predictive accuracy of $94 \%$ (figure $3 \mathrm{~B}$ ). These results demonstrate that the plasma metabolome of omnivores, relative to vegans, particularly reflects the differential intake of meat and fat, demonstrating a direct effect of diet on the host metabolome. However, a few metabolites predominant in the plasma of omnivores (see online supplementary able S3) are cometabolites generated with contributions from both the host and the gut microbiota. These include monomethyl branched-chain fatty acids (mmBCFAs), such as 13-methylmyristate and 15-methylpalmitate. mmBCFAs can be derived from meat and dairy consumption, ${ }^{22}$ produced endogenously from branchedchain amino acids (ie, valine, leucine and isoleucine ${ }^{23}$ or be synthesised by bacteria and incorporated into bacterial cell membranes. $^{24}$

Targeted quantification of urinary metabolites derived from metabolism by the gut microbiota also reveals a significant separation between omnivores and vegans (figure $3 \mathrm{C}$ ). The detection of these metabolites in both the urine and the plasma (see online supplementary table S3) reflects the dynamic relationship regulating plasma levels of these metabolites that are absorbed by the host after production by the gut microbiota with subsequent excretion in the urine.

In contrast to diet, the microbiota composition was not strongly associated with the plasma metabolome. In pairwise associations of microbial taxa and metabolites after adjustment for vegan versus omnivore diet, the strongest association was for Barnesiellaceae with cystine $(\mathrm{p}=0.00002, \mathrm{q}=0.24)$; no unique bacterial taxa were significantly associated with individual metabolite levels after adjustment for multiple comparisons. In conjunction with the modest effect of these diets on the composition of the gut microbiota (figure 2), this minimal association is consistent with a dominant role of diet in determining the host metabolome.

\section{Diet-dependent metabolites produced by the gut microbiota of vegans.}

Vitamins and other plant-based products such as ascorbate, xanthine metabolites and products of benzoate metabolism were elevated in the plasma metabolome of vegans relative to omnivores. Several of these reflect the plant-based diet of vegans (figure $1 \mathrm{~B}$ and see online supplementary table S1). Twenty-eight metabolites were more abundant in vegans with a $\mathrm{q}$ value $<0.05$. Approximately one-third of these metabolites are composed of products produced by the gut microbiota (see online supplementary table S3: marked in gray). ${ }^{25}$ One example is the benzoate metabolism of plant polyphenolic compounds (figure 4). Grains, nuts and berries, prominent in the vegan diet, contain phenolic phytochemicals such as hydroxybenzoic acids, hydroxycinnamic acids, flavonoids and condensed tannins. Bioavailability of phenolic compounds is limited due to covalent linkage to the plant cell walls. Gut absorption, enhanced by the enzymatic activity of the gut microbiota, reduces these polyphenols into smaller phenolic compounds. Several metabolites suggestive of chlorogenic acid bacterial metabolism, including hippurate, catechol sulfate and 3-hydroxyhippurate, were increased in vegans, as were other likely polyphenolic gut cometabolites such as 4-hydroxyhippurate, ${ }^{26}$ 4-ethylphenylsulfate (identified in the RF classification, figure 3B) and 4-vinylphenol sulfate, whose metabolic origins are less well established. ${ }^{27} 28$ 
A
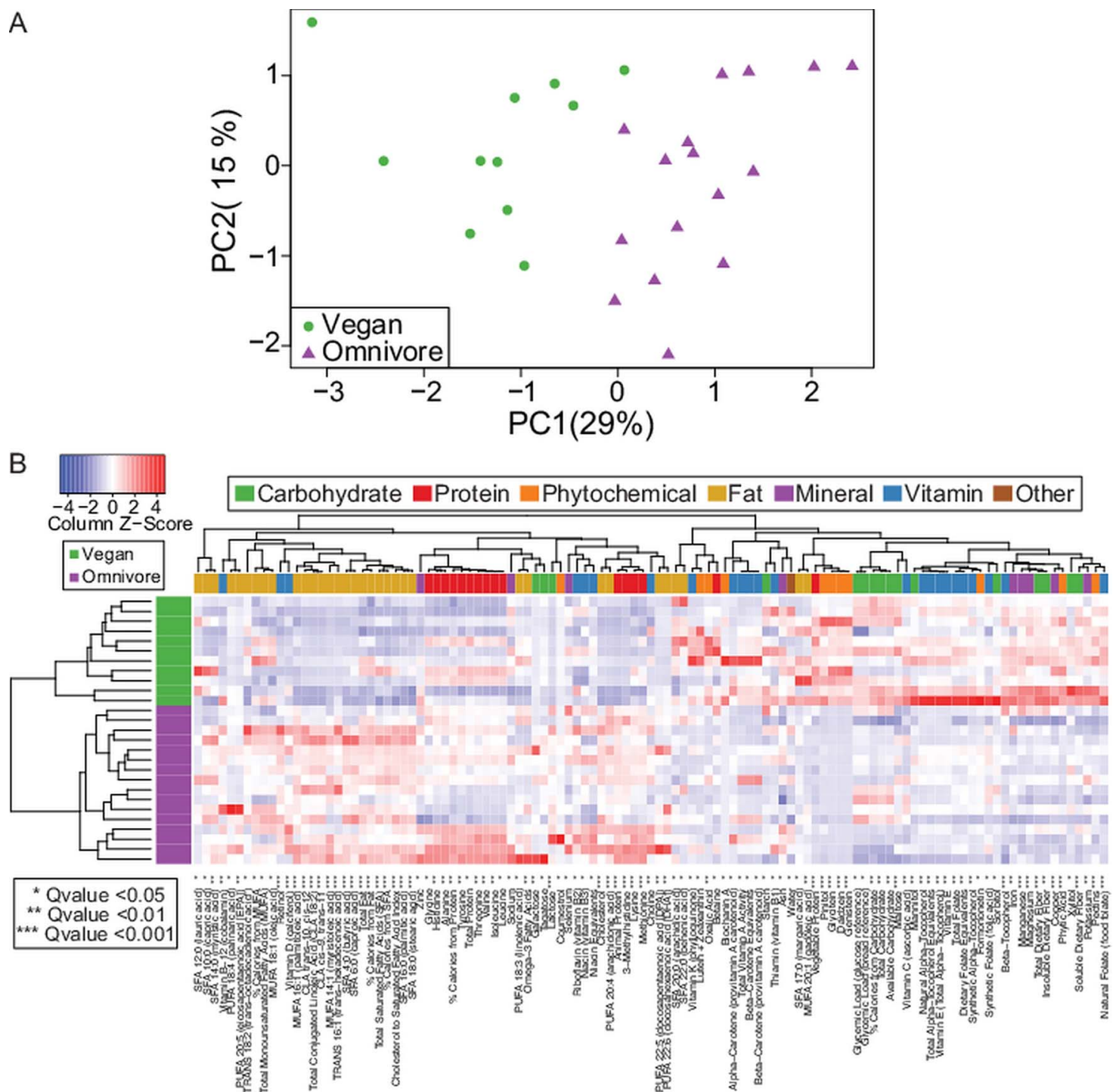

Figure 1 Dietary consumption comparison between omnivores and vegans residing in an urban US environment. (A) Principle component analysis of dietary micronutrient consumption in omnivores versus vegans. (B) Heat map dietary micronutrients in omnivores (purple) versus vegans (green) colour coded by seven major nutrient categories. Red=higher abundance, blue=lower abundance, $\mathrm{p}$ values as indicated by the number of asterisks. $\mathrm{PC}$, principle component.

\section{A plant-based vegan diet does not alter faecal short chain fatty acid or breath methane levels.}

Individuals living in Western societies have lower levels of faecal SCFA and methane production than individuals from traditional agrarian societies. ${ }^{29}$ These differences have been attributed to increase in polysaccharide-degrading microbiota combined with an increased consumption of indigestible fibre, which is a substrate for bacterial fermentation. ${ }^{2}$ However, despite the consumption of a plant-based diet in vegans (figure $1 \mathrm{~B}$ and see online supplementary table S1), quantification of faecal SCFA levels by proton NMR (see online supplementary table S4) and methane production by breath testing (see online supplementary table S5) revealed no difference between vegans and omnivores in our cohort.

The residence in an agrarian society is associated with a gut microbiota differing from individuals residing in Western societies. $^{2} 33031$ Genomic representation for fermentative pathways, based on a PICRUSt analysis, suggested a greater capacity for SCFA production in Africans in Burkina Faso (BF) than residents in the European Union (EU). Predicted gene representation for glycan-degrading enzymes showed differences for the BF versus EU subjects (49 out of 76 significant; 'glycan biosynthesis and metabolism' and 'carbohydrate digestion and absorption' greater in BF, $\mathrm{p}=1 \times 10^{-7}$ and $1 \times 10^{-8}$, respectively, by Welch two sample $t$ test), whereas a similar analysis did not show a difference for omnivores versus vegans studied here ( $p>0.18$ for both comparisons). Nevertheless, the vegan diet more resembled residents in BF than the EU-vegans showing a greater intake of fibre, carbohydrate and starch, with lower intake of energy, fat, protein and sugars (see online supplementary table S4). Together, these results suggest that faecal SCFA levels, determined by bacterial fermentation, are regulated by both substrate abundance provided by diet and the bacterial lineages comprising the gut microbiota. ${ }^{32}$

We confirmed these findings in a CAFÉ, whereby omnivorous healthy human subjects were randomised to consume a highfibre/low-fat or low-fibre/high-fat diet for 10 days. ${ }^{6}$ To achieve these dietary goals with no change in protein or energy intake, the intake of sugars and carbohydrates was manipulated concurrent with the desired changes in fat and fibre intake. Colonic 
A

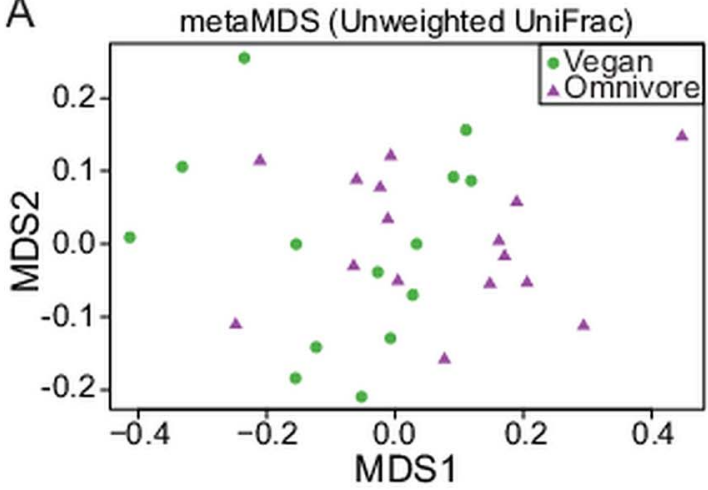

C

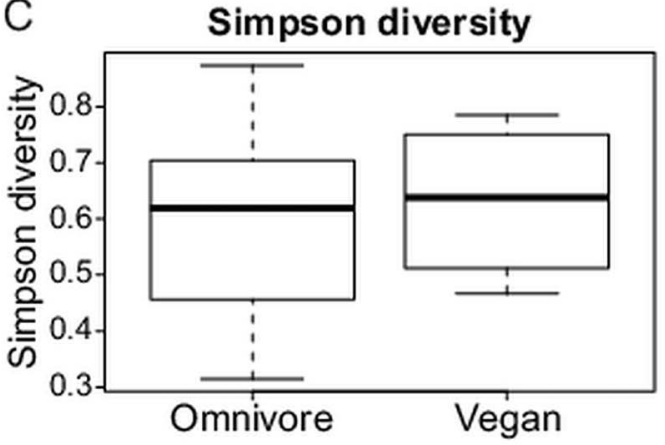

B

metaMDS (Weighted UniFrac)

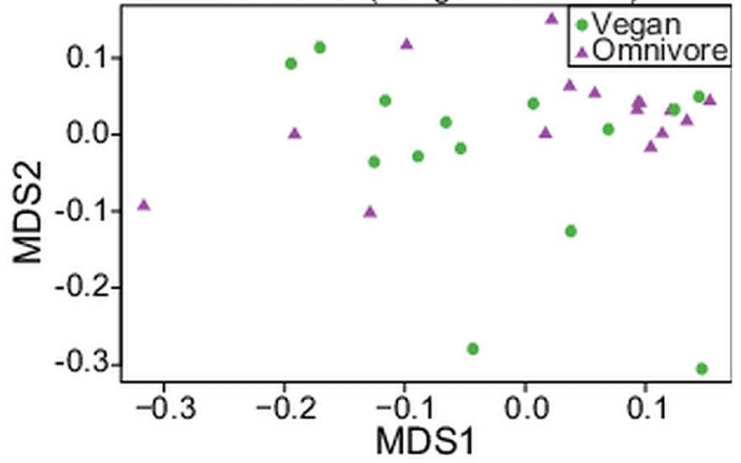

Figure 2 The composition of the gut microbiota in omnivore versus vegans show very modest differences. Multidimensional scaling (MDS) analysis of gut microbiota composition in omnivore versus vegans determined by $16 \mathrm{~S}$ rRNA gene sequencing by $(\mathrm{A})$ unweighted UniFrac distance (PERMANOVA $p=0.007$ ), (B) weighted UniFrac distance (PERMANOVA $p=0.15$ ). (C) Comparison of Simpson diversity index in omnivores versus vegans $(p=0.53)$.

transit time was longer in the group consuming the low fibre relative to the high-fibre diet (see online supplementary figure S3). ${ }^{6}$ Despite significant increases in fibre, starch, sugar and total carbohydrate consumption, there were only very modest effects of diet on the composition of the gut microbiota ${ }^{6}$ and no significant effect on the levels of faecal SCFAs (see online supplementary table S4). Furthermore, the high level of intersubject variability in faecal SCFA levels at baseline in this CAFÉ was not reduced in either the high-fibre or the low-fibre group despite the consumption of an identical diet for 10 days within each group ${ }^{6}$ (see online supplementary figure S4) suggesting that intersubject differences in gut microbiota composition may determine SCFA levels. Indeed, consumption of identical diets in the CAFÉ did not reduce gut microbiota intersubject variability nor did it lead to consistent alterations in the gut microbiota among subjects on the same diet. ${ }^{6}$

\section{Diet and the gut microbiota in the production of phytoestrogens}

Phytoestrogens are hypothesised to have numerous health benefits. ${ }^{33}$ Equol, a phytoestrogen, is a byproduct of metabolism of isoflavones found in high concentrations in soy-based foods. While only $30 \%$ of adults in Western populations are able to produce equol from soy isoflavones, nearly $60 \%-70 \%$ of residents in Asia are equol producers. ${ }^{34}$ The omnivores consumed relatively little phytoestrogen precursors for equol generation (daidzein and geninstein) and none had detectable equol in plasma. In contrast, vegans consumed much more phytoestrogen precursors for equol generation (figure 5), yet only $40 \%$ of the vegans had detectable equol in plasma $(p=0.006)$. Among vegans, consumption of isoflavones was slightly higher among those with detectable plasma equol (figure 5) but none of these trends were statistically significant (daidzein $\mathrm{p}=0.22$; geninstein $\mathrm{p}=0.21$ ). Thus, these data also emphasise that both exposure to substrates and microbial metabolic capacity jointly determine the cometabolites observed in plasma.

\section{DISCUSSION}

The composition of the gut microbiota has been linked to residence in Western versus agrarian societies ${ }^{2} 33035$ and to differences in diet. ${ }^{2} 67$ Perhaps, the largest impact of diet has been observed when carbohydrates were eliminated from the diet, ${ }^{36}$ though exclusion of all carbohydrates is not sustainable or beneficial to health in humans. Controlled feeding studies in humans using less extreme differences in diet suggest that the effects on gut bacterial communities can be much more modest. ${ }^{6} 37$ Veganism is one of the most extreme dietary patterns that is sustainable for extended periods in Western countries. Here, we compared urban dwelling vegans and omnivores from the same US environment, revealing that the considerable dietary differences correlated with large variations in the metabolome, including cometabolites produced by the gut microbiota, but that differences in bacterial community composition were modest. This suggests that if the significant differences in gut microbiota composition in globally distinct human populations $^{2} 3{ }^{31}$ are due to diet, such differences may take several generations to evolve or require very early life exposures. Alternatively, the differences in microbiota composition may be, in part, due to environmental factors independent of diet.

In our study, major differences in the metabolome included increased levels of cometabolites that result from metabolism of plant-derived products by the gut microbiota among vegans and increased levels of lipids and amino acids derived from the consumption of animal products among omnivores. ${ }^{26}$ The gut 


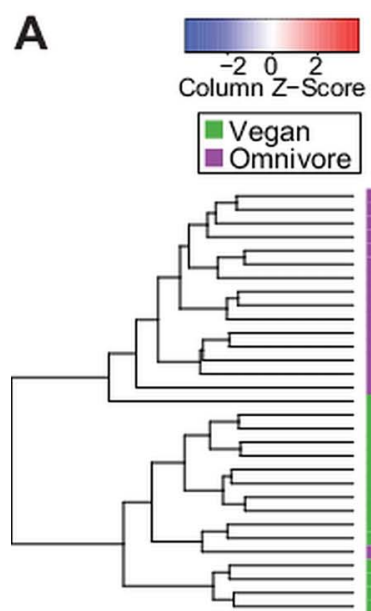

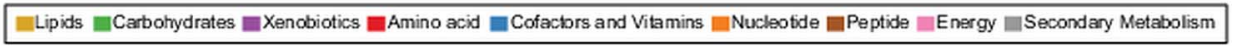
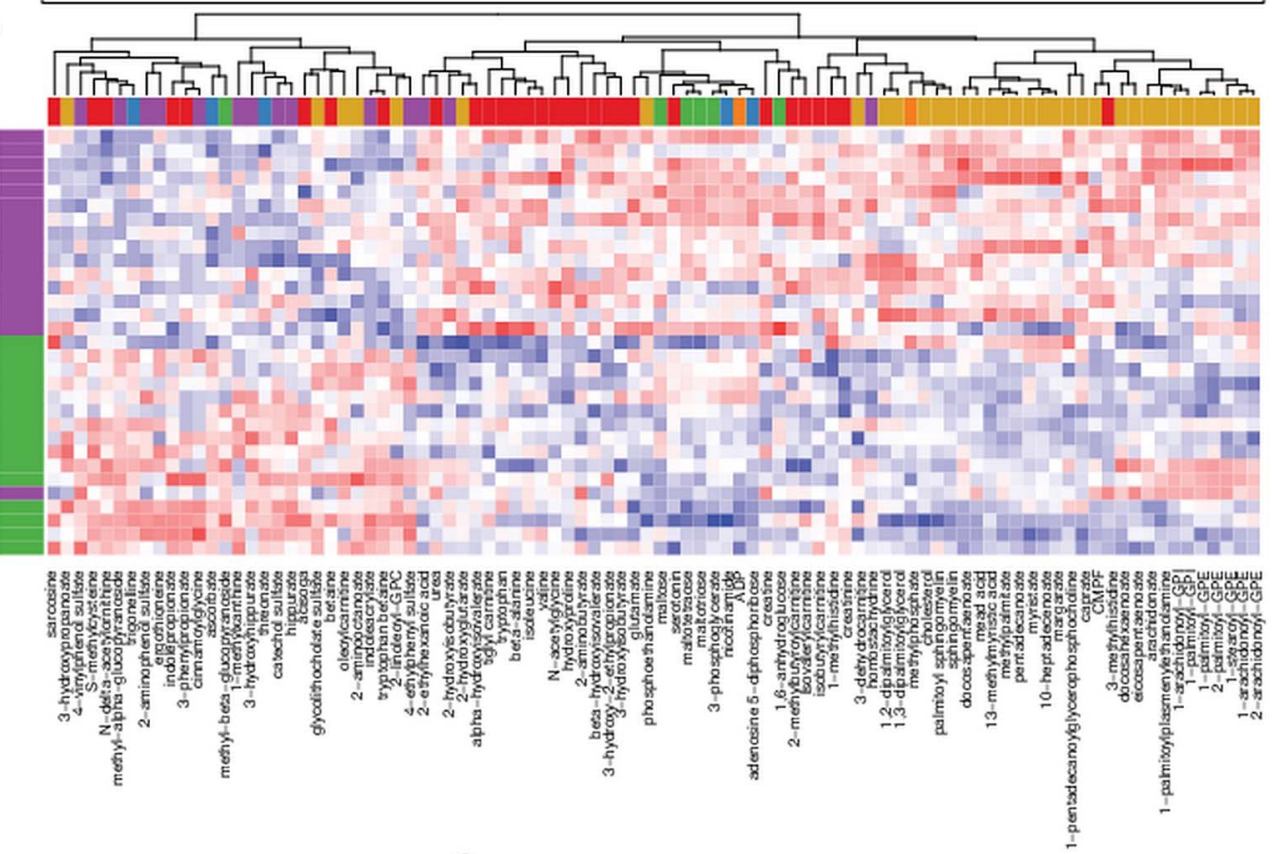

B

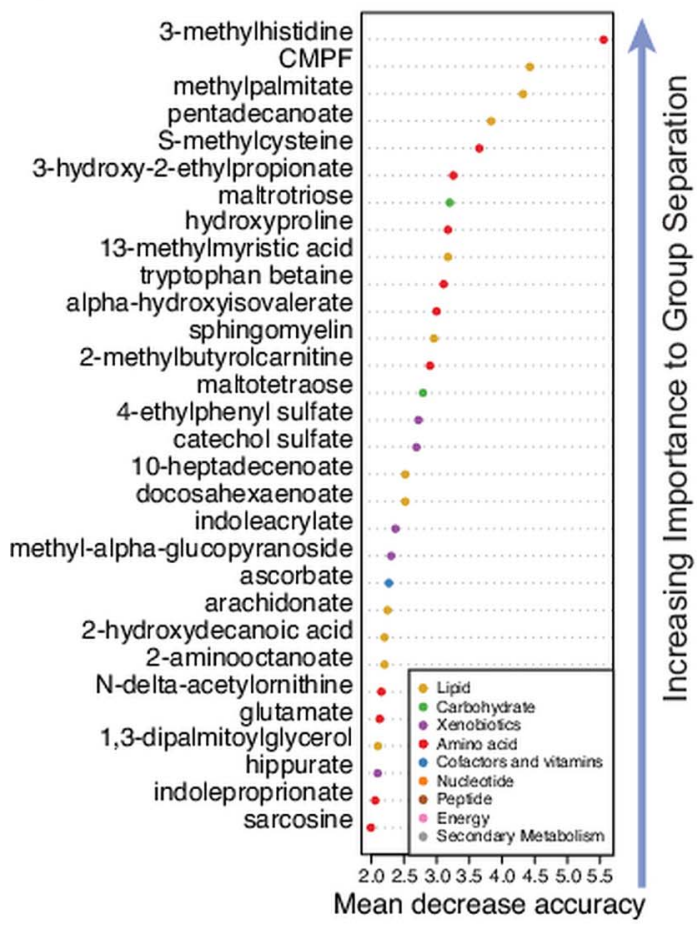

C

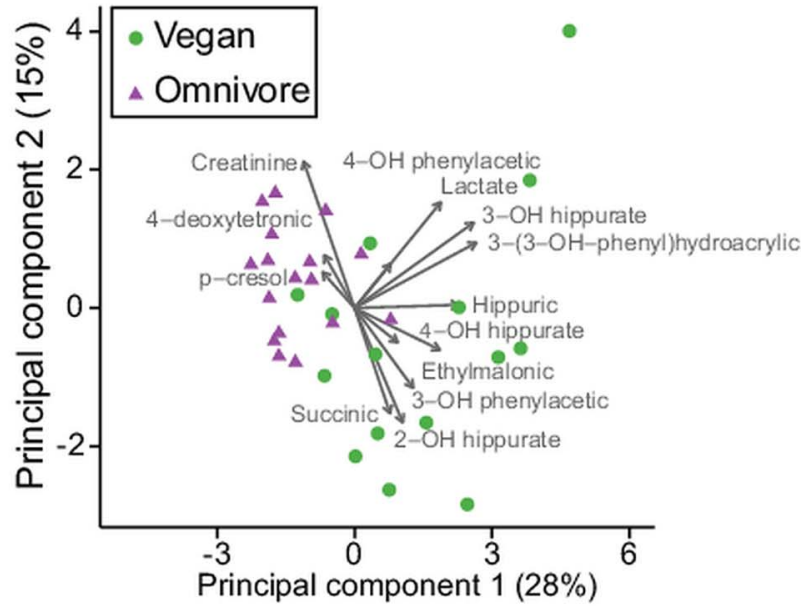

Figure 3 Comparison of plasma metabolites in omnivores versus vegans. (A) Hierarchical clustering correlating levels of plasma metabolites between omnivores versus vegans visualised as a heatmap. Red=higher abundance, blue=lower abundance. (B) Random forest classification of 30 plasma metabolites, categorised into seven areas (colour coded), capable of distinguishing an omnivore from a vegan with a predictive accuracy of $94 \%$. (C) Targeted quantification of urinary metabolites in omnivores and vegans visualised in a principal components analysis biplot that displays the correlation of each sample with the metabolite descriptors. Metabolite descriptors are represented by arrows pointing in the direction of maximum correlation with the samples.

microbiota, therefore, contributes more prominently to the plasma metabolome of vegans than omnivores. Although only a modest number of these metabolites are known products of gut microbial metabolism because the eukaryotic genome is incapable of producing them, studies in rodents suggest that the contribution of the gut microbiota to the host metabolome may be larger, numbering in the hundreds of chemical species. ${ }^{38}$ Plant-based diets are well known to serve as substrates by which the gut microbiota can produce metabolites that are subsequently absorbed by the host. A previous study comparing the urinary metabolome of omnivores and lactovegetarians by proton NMR spectroscopy identified approximately a dozen 

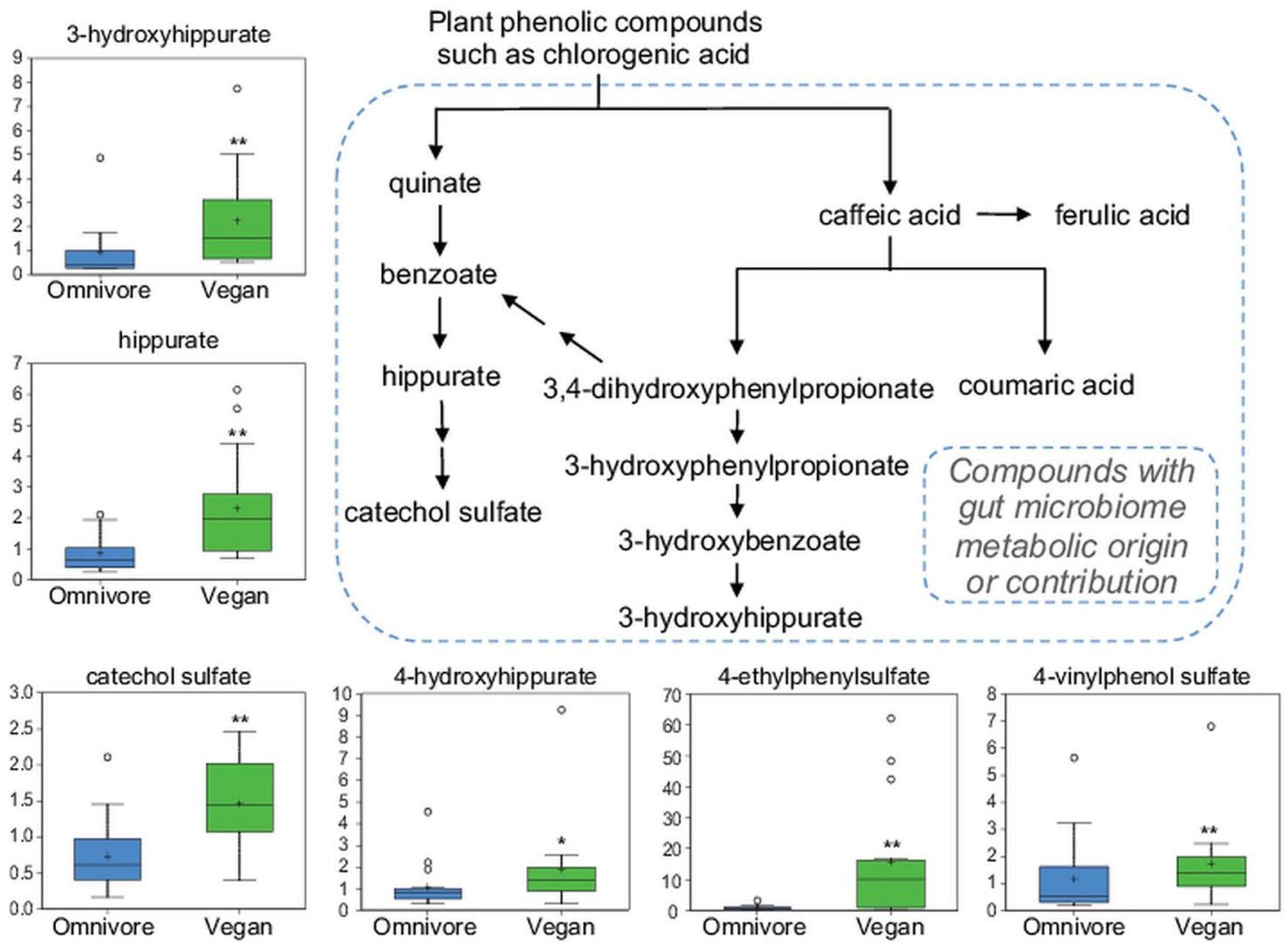

Figure 4 Plasma levels of plasma metabolites in omnivores and vegans derived from gut microbiota metabolism of plant polyphenolic compounds. ${ }^{*} p<0.1,{ }^{*} p<0.05$

metabolites that distinguished subjects in these two groups. ${ }^{39}$ Our results extend this observation by showing the co-occurrence of differentially expressed metabolites in both the plasma and urine of omnivores and vegans demonstrating that the plasma metabolome is regulated by both metabolite production as well as excretion, a balance that is hypothesised to have important health implications. For example, the decreased renal clearance and subsequent elevated plasma levels of certain metabolites delivered to the host by the gut microbiota, such as p-cresol and trimethylamine oxide, may play a role in the development of comorbidities in patients with chronic kidney disease. ${ }^{10} 1140-42$

The very modest difference between the gut microbiota of omnivores relative to vegans juxtaposed to the significantly enhanced dietary consumption of fermentable plant-based foods in the latter provides a unique opportunity to examine the impact of gut microbiota composition on the production of metabolites such as SCFAs. Faecal SCFA levels in residents of agrarian societies are very high relative to those living in Westernised societies ${ }^{2} 31$ and correlate with increased faecal bacterial numbers, yet there is little increase in faecal SCFA levels in omnivorous humans residing in Western societies when receiving dietary supplements of fermentable carbohydrates ${ }^{43}$ (reviewed in ref. 44)-findings similar to our results comparing omnivores and vegans and our CAFÉ. By contrast, significant reduction of dietary carbohydrates in omnivores leads to much lower levels of faecal SCFAs and decreased bacterial numbers. $^{3645}$ We propose the following model to explain these observations by considering the relationship between fermentable substrate abundance in diet to product generation by fermentation within the context of gut microbiota composition (figure 6). The coevolution of the gut microbiota with its herbivorous mammalian host has led to a microbial community capable of delivering high levels of SCFAs to the host on a plant-based diet. ${ }^{44}$ Taxonomic representation of a fermentative signature predominant in nonhuman herbivorous mammals ${ }^{1}$

Figure 5 Association between the consumption of dietary substrates used by the gut microbiota to produce equol showing the standardised intake of diadzein and geneistein in omnivores and vegans with and without detectable plasma levels of equol. 


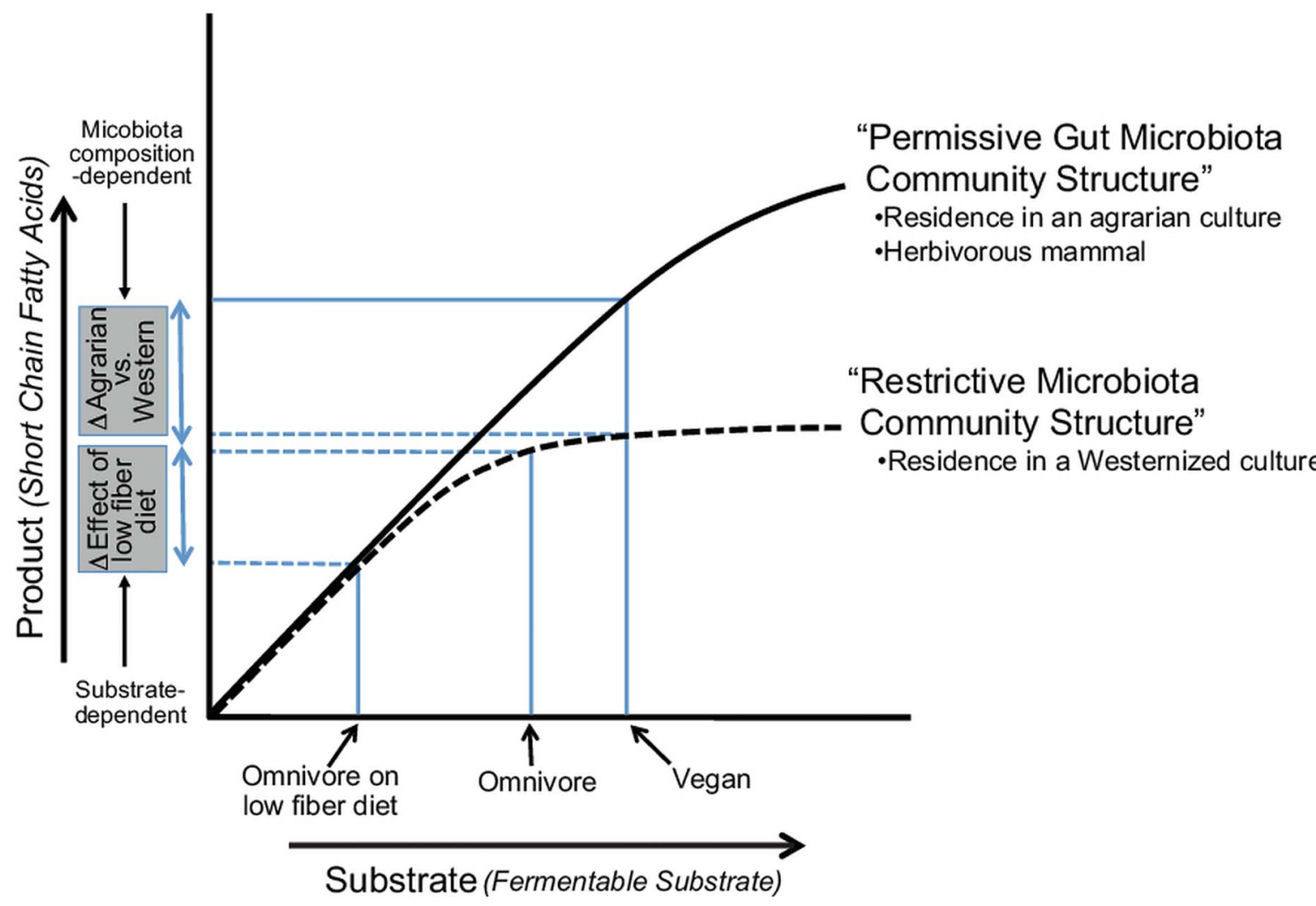

Figure 6 Model describing the relationship between substrate abundance and gut microbiota product formation and its dependence on gut microbiota composition using the consumption of fermentable carbohydrates and the production of short chain fatty acids (SCFAs) as an example. Herbivorous mammals and humans residing in agrarian societies, with a predominance of gut bacteria capable of producing SCFAs through fermentation, have a 'permissive' community structure of their gut microbiota and exhibit substrate-dependent production of product that is linear. By contrast, residence of humans in Westernised societies have a 'restrictive community structure' where additional substrate delivery leads to a minimal increase in product formation due to saturation. However, both community structures are substrate-dependent whereby a decrease in substrate consumption (ie, omnivores on a low carbohydrate/fibre diet) will lead to a decrease in product formation (SCFAs).

together with society-dependent studies identifying specific bacteria taxa capable of high levels of fermentation supports this notion. ${ }^{32}$ Similarly, the distinctive gut microbiota in human residents of agrarian societies facilitates high SCFA production associated with the consumption of a plant-based diet. ${ }^{2} 31$ The enrichment of genera such as Prevotella, whose relatives found in the rumen are rich in xylanase, carboxymethylcellulase and endoglucanase, ${ }^{2} 46$ may establish a 'permissive' gut microbiota capable of producing high levels of SCFAs on a plant-based diet.

By contrast, we propose that residents in Westernised societies have a 'restrictive' gut microbiota composition not capable of producing high levels of SCFAs when high levels of fermentable plant products are consumed. This may be due to the absence of certain 'keystone' species whose activities are required to initiate degradation of these recalcitrant substrates. Lack of the keystone species Ruminococcus bromii has been proposed to explain the incomplete fermentation of resistant starch in some human volunteers ${ }^{47}$ and it seems highly likely that keystone species exist for the many types of plant cell walls represented in Western and agrarian diets, but remain to be identified. Indeed, our results suggest that the long-term consumption of a plant-based diet in industrialised nations, at least as represented by the vegans studied here, does not lead to a gut microbiota that is strongly divergent from that of omnivores. We confirm these findings in a CAFÉ comparing a high-fibre versus low-fibre diet where there are minimal alterations in gut microbiota composition $^{6}$ and no effect on levels of SCFAs. Other factors that may alter SCFA production in omnivores versus vegans that are independent of bacterial composition such as methanogenesis ${ }^{48}$ and gut transit ${ }^{49}$ were excluded by showing that there is no difference in methane production and more rapid transit on a high-fibre diet. Nevertheless, substrate abundance is also critical, as demonstrated in studies showing that a reduction of dietary fibre significantly lowers faecal SCFAs in humans (figure 6). ${ }^{36} 45$ Thus, both diet-derived substrates and the gut microbes present seem to be determinants of the cometabolites produced.

Our model also provides an explanation for the relationship between the omnivore versus vegan diet and plasma levels of S-equol, a non-steroidal oestrogen that may exhibit health benefits, produced by gut microbiota metabolism of isoflavones rich in soy foods, such as daidzein and genistein. ${ }^{33}$ Germ-free animals and infants aged $<4$ months do not produce $S$-equol in response to soy consumption. ${ }^{50} 51$ Although almost every animal species can produce equol when consuming soycontaining diet, ${ }^{33}$ humans differ in that only about $30 \%$ of the Western population can produce S-equol after consuming soy isoflavones, whereas approximately $60 \%$ of adults in Asian populations are equol producers, a group where soy is more regularly consumed. ${ }^{34}$ The basis for the variability in producing S-equol is currently unknown but may be due to differential membership in the gut microbiota. ${ }^{10} 52$ However, the observation that equol production is a stable trait over time ${ }^{53}$ and that the consumption of soy does not convert a non-equol producer into an equol producer ${ }^{33}$ suggest that the ability to produce 
equol is more a consequence of colonisation associated with residence in Asian versus Western societies than by diet. Only $40 \%$ of vegans in our study had detectable plasma equol levels, reflecting a Westernised influence on the trait and emphasising that both bacterial lineages present and substrate availability likely account for differential equol production. Indeed, similar to the association between extremely low carbohydrate diets and the significant reduction in faecal SCFAs, ${ }^{3645}$ omnivores in our study consumed low levels of the two soy-based substrates and none had detectable levels of plasma equol.

In summary, agrarian diets have been associated with better health due to incompletely characterised mechanisms. Bacterial metabolites were more abundant while lipid and amino acid metabolites were less abundant in the plasma metabolome of vegans, which may provide a health benefit relative to omnivores. A plant-based diet may increase delivery of beneficial bacterially produced metabolites, such as equol, or decrease the production of a deleterious metabolite such as trimethylamine. ${ }^{11}$ Our results support a greater role for diet as a substrate that influences the bacterial metabolome than as a factor that regulates gut bacterial community membership. However, the production of some metabolites may be constrained by the composition of the gut microbiota, which may be more a consequence of residence in Western, African or Asian societies than by diet. An example of a culturally associated environmentally determined gut microbiota trait is a specific glycoside hydrolase, capable of degrading porphyran that is abundant in red algae, frequent in the gut microbiome of the Japanese population but absent in Northern American individuals. ${ }^{54}$ Thus, consideration of gut microbiota composition may be important when developing a 'prebiotic' approach to treat disease and/or maintain health by delivering specific substrates for bacterial conversion into beneficial metabolites. Such prebiotics may demonstrate varying levels of efficacy in culturally distinct human populations. Integrating information about the composition of the gut microbiome with the delivery of substrates focused on metabolite production should help make possible both improved diets and the 'next-generation' prebiotics, probiotics, synbiotics and dietary supplements for maintaining health and treating disease.

\section{Author affiliations}

${ }^{1}$ Division of Gastroenterology, Perelman School of Medicine, The University of Pennsylvania, Philadelphia, Pennsylvania, USA

${ }^{2} S c h o o l$ of Nursing, Perelman School of Medicine, The University of Pennsylvania, Philadelphia, Pennsylvania, USA

${ }^{3}$ Departments of Biostatistics \& Epidemiology, Perelman School of Medicine, The University of Pennsylvania, Philadelphia, Pennsylvania, USA

${ }^{4}$ Divisions of Endocrinolgy, The Children's Hospital of Philadelphia, Philadelphia, Pennsylvania, USA

${ }^{5}$ Department of Microbiology, Perelman School of Medicine, The University of Pennsylvania, Philadelphia, Pennsylvania, USA

${ }^{6}$ Department of Gastroenterology, Hepatology, and Nutrition, The Children's Hospital of Philadelphia, Philadelphia, Pennsylvania, USA

${ }^{7}$ Department of Pharmacology, Perelman School of Medicine, The University of Pennsylvania, Philadelphia, Pennsylvania, USA

${ }^{8}$ Microbiology Group, Rowett Institute of Nutrition and Health, University of Aberdeen, Aberdeen, UK

${ }^{9}$ Department of Pathology and Laboratory Medicine, The Children's Hospital of Philadelphia, Philadelphia, Pennsylvania, USA

Acknowledgements This work was supported by Project NIH UH2/3 DK083981 and administrative supplement 04S1 (GDW, FDB and JDL), Scottish Government Food, Land and People programme (HJF), The Molecular Biology Core of the Penn Center for the Molecular Studies in Digestive and Liver Diseases (P30 DK050306), The Joint Penn-CHOP Center for Digestive, Liver and Pancreatic Medicine and Penn Vegan Society.

Contributors GDW, JDL and FDB were responsible for the overall concept, design and supervision of the study as well as writing the manuscript. CC, HJF and LGA provided nutritional analyses. SAS, RDS, AMW, MJB and LGA performed experiments. DCM, LN, EG and JS recruited subjects and collected biospecimens. $E Z C, K B, C C$ and $H L$ performed computational and statistical analyses.

Funding NIH UH2/3 DK083981 and administrative supplement 04S1 as well as the Scottish Government Food, Land and People Programme.

Competing interests None.

Ethics approval University of Pennsylvania Institutional Review Board.

Provenance and peer review Not commissioned; externally peer reviewed.

\section{REFERENCES}

1 Ley RE, Hamady M, Lozupone $\mathrm{C}$, et al. Evolution of mammals and their gut microbes. Science 2008;320:1647-51.

2 De Filippo C, Cavalieri D, Di Paola M, et al. Impact of diet in shaping gut microbiota revealed by a comparative study in children from Europe and rural Africa. Proc Natl Acad Sci U S A 2010;107:14691-6.

3 Yatsunenko T, Rey FE, Manary MJ, et al. Human gut microbiome viewed across age and geography. Nature 2012;486:222-7.

4 Muegge BD, Kuczynski J, Knights $D$, et al Diet drives convergence in gut microbiome functions across mammalian phylogeny and within humans. Science 2011;332:970-4

5 Hildebrandt MA, Hoffmann C, Sherrill-Mix SA, et al. High-fat diet determines the composition of the murine gut microbiome independently of obesity. Gastroenterology 2009;137:1716-24 e1-2.

6 Wu GD, Chen J, Hoffmann C, et al. Linking long-term dietary patterns with gut microbial enterotypes. Science 2011;334:105-8.

7 Claesson MJ, Jeffery IB, Conde S, et al. Gut microbiota composition correlates with diet and health in the elderly. Nature 2012;488:178-4.

8 Cotillard A, Kennedy SP, Kong LC, et al. Dietary intervention impact on gut microbial gene richness. Nature 2013;500:585-8.

9 Holmes E, Li JV, Marchesi JR, et al. Gut microbiota composition and activity in relation to host metabolic phenotype and disease risk. Cell Metab 2012;16:559-64.

10 Wang Z, Klipfell E, Bennett BJ, et al. Gut flora metabolism of phosphatidylcholine promotes cardiovascular disease. Nature 2011;472:57-63.

11 Koeth RA, Wang Z, Levison BS, et al. Intestinal microbiota metabolism of $\mathrm{L}$-carnitine, a nutrient in red meat, promotes atherosclerosis. Nat Med 2013;19:576-85

12 Qin J, Li R, Raes J, et al. A human gut microbial gene catalogue established by metagenomic sequencing. Nature 2010;464:59-65.

13 Wu GD, Lewis JD, Hoffmann C, et al. Sampling and pyrosequencing methods for characterizing bacterial communities in the human gut using 165 sequence tags. BMC Microbiol 2010;10:206.

14 Hoffmann C, Minkah N, Leipzig J, et al. DNA bar coding and pyrosequencing to identify rare HIV drug resistance mutations. Nucleic Acids Res 2007;35:e91.

15 Hamady M, Walker JJ, Harris JK, et al. Error-correcting barcoded primers for pyrosequencing hundreds of samples in multiplex. Nature Methods 2008;5:235-7.

16 Caporaso JG, Kuczynski J, Stombaugh J, et al. QIIME allows analysis of high-throughput community sequencing data. Nat Methods 2010;7:335-6.

17 Jones PM, Bennett MJ. Urine organic acid analysis for inherited metabolic disease using gas chromatography-mass spectrometry. Methods Mol Biol 2010;603:423-1.

18 Weljie AM, Newton J, Mercier P, et al. Targeted profiling: quantitative analysis of 1H NMR metabolomics data. Anal Chemistry 2006:78:4430-42.

19 Riordan SM, Mclver CJ, Walker BM, et al. The lactulose breath hydrogen test and small intestinal bacterial overgrowth. Am J Gastroenterol 1996;91:1795-803.

20 Langille MG, Zaneveld J, Caporaso JG, et al. Predictive functional profiling of microbial communities using 16S rRNA marker gene sequences. Nat Biotechnol 2013;31:814-21.

21 Craig WJ. Health effects of vegan diets. Am J Clin Nutr 2009;89:1627S-33S.

22 Ran-Ressler RR, Sim D, O'Donnell-Megaro AM, et al. Branched chain fatty acid content of United States retail cow's milk and implications for dietary intake. Lipids 2011:46:569-76

23 Oku H, Yagi N, Nagata J, et al. Precursor role of branched-chain amino acids in the biosynthesis of iso and anteiso fatty acids in rat skin. Biochimica et Biophysica Acta 1994;1214:279-87.

24 Kniazeva M, Crawford QT, Seiber M, et al. Monomethyl branched-chain fatty acids play an essential role in Caenorhabditis elegans development. PLOS Biol 2004;2:E257.

25 Kumps A, Duez P, Mardens Y. Metabolic, nutritional, iatrogenic, and artifactual sources of urinary organic acids: a comprehensive table. Clinical Chemistry 2002;48:708-17.

26 Heinzmann SS, Merrifield CA, Rezzi S, et al. Stability and robustness of human metabolic phenotypes in response to sequential food challenges. J Proteome Res 2012;11:643-55.

27 Silva I, Campos FM, Hogg T, et al. Wine phenolic compounds influence the production of volatile phenols by wine-related lactic acid bacteria. J App/ Microbiol 2011;111:360-70. 
28 Jo YJ, Cho IH, Song CK, et al. Comparison of fermented soybean paste (Doenjang) prepared by different methods based on profiling of volatile compounds. J Food Sci 2011;76:C368-79.

29 Levitt MD, Furne JK, Kuskowski M, et al. Stability of human methanogenic flora over 35 years and a review of insights obtained from breath methane measurements. Clin Gastroenterol Hepatol 2006;4:123-9.

30 Lin A, Bik EM, Costello EK, et al. Distinct distal gut microbiome diversity and composition in healthy children from Bangladesh and the United States. PLOS ONE 2013;8:e53838.

31 Ou J, Carbonero F, Zoetendal EG, et al. Diet, microbiota, and microbial metabolites in colon cancer risk in rural Africans and African Americans. Am J Clin Nutr 2013;98:111-20.

32 Russell WR, Hoyles L, Flint HJ, et al. Colonic bacterial metabolites and human health. Curr Opin Microbiol 2013;16:246-54.

33 Atkinson C, Frankenfeld CL, Lampe JW. Gut bacterial metabolism of the soy isoflavone daidzein: exploring the relevance to human health. Exp Biol Med 2005;230:155-70.

34 Magee PJ. Is equol production beneficial to health? Proc Nutr Soc 2011;70:10-18

35 Faust K, Sathirapongsasuti JF, Izard J, et al. Microbial co-occurrence relationships in the human microbiome. PLoS Comput Biol 2012;8:e1002606.

36 David LA, Maurice CF, Carmody RN, et al. Diet rapidly and reproducibly alters the human gut microbiome. Nature 2014;505:559-63.

37 Walker AW, Ince J, Duncan SH, et al. Dominant and diet-responsive groups of bacteria within the human colonic microbiota. ISME J 2011;5:220-30.

38 Zheng $X$, Xie G, Zhao A, et al. The footprints of gut microbial-mammalian co-metabolism. J Proteome Res 2011;10:5512-22.

39 Xu J, Yang S, Cai S, et al. Identification of biochemical changes in lactovegetarian urine using $1 \mathrm{H}$ NMR spectroscopy and pattern recognition. Anal Bioanal Chem 2010;396:1451-63.

40 Bain MA, Faull R, Fornasini G, et al. Accumulation of trimethylamine and trimethylamine-N-oxide in end-stage renal disease patients undergoing haemodialysis. Nephrol Dial Transplant 2006;21:1300-4.

41 Hage FG, Venkataraman R, Zoghbi GJ, et al. The scope of coronary heart disease in patients with chronic kidney disease. J Am Coll Cardiol 2009;53:2129-40.
42 Bammens $B$, Evenepoel $P$, Keuleers $H$, et al. Free serum concentrations of the protein-bound retention solute $\mathrm{p}$-cresol predict mortality in hemodialysis patients. Kidney Int 2006;69:1081-7.

43 Scheppach WM, Fabian CE, Kasper HW. Fecal short-chain fatty acid (SCFA) analysis by capillary gas-liquid chromatography. Am J Clin Nutr 1987;46:641-6.

44 den Besten $G$, van Eunen K, Groen AK, et al. The role of short-chain fatty acids in the interplay between diet, gut microbiota, and host energy metabolism. J Lipid Res 2013:54:2325-40.

45 Duncan SH, Belenguer A, Holtrop G, et al. Reduced dietary intake of carbohydrates by obese subjects results in decreased concentrations of butyrate and butyrate-producing bacteria in feces. Appl Environ Microbiol 2007;73: 1073-8.

46 White BA, Lamed R, Bayer EA, et al. Biomass Utilization by Gut Microbiomes. Annu Rev Microbiol 2014;68:279-6.

47 Ze $X$, Duncan $\mathrm{SH}$, Louis $\mathrm{P}$, et al. Ruminococcus bromii is a keystone species for the degradation of resistant starch in the human colon. ISME J 2012;6:1535-43.

48 Samuel BS, Gordon Jl. A humanized gnotobiotic mouse model of host-archaeal-bacterial mutualism. Proc Natl Acad Sci USA 2006;103:10011-16.

49 El Oufir L, Flourie B, Bruley des Varannes $S$, et al. Relations between transit time, fermentation products, and hydrogen consuming flora in healthy humans. Gut 1996;38:870-7

50 Bowey E, Adlercreutz H, Rowland I. Metabolism of isoflavones and lignans by the gut microflora: a study in germ-free and human flora associated rats. Food Chem Toxicol 2003;41:631-6.

51 Setchell KD, Zimmer-Nechemias L, Cai J, et al. Exposure of infants to phyto-oestrogens from soy-based infant formula. Lancet 1997;350:23-7.

52 Craciun S, Balskus EP. Microbial conversion of choline to trimethylamine requires a glycyl radical enzyme. Proc Natl Acad Sci USA 2012;109:21307-12.

53 Frankenfeld CL, Atkinson C, Thomas WK, et al. High concordance of daidzein-metabolizing phenotypes in individuals measured 1 to 3 years apart. $\mathrm{Br} \mathrm{J}$ Nutr 2005;94:873-6.

54 Hehemann JH, Correc G, Barbeyron T, et al. Transfer of carbohydrate-active enzymes from marine bacteria to Japanese gut microbiota. Nature 2010;464:908-12. 